\title{
Windowing Design and Performance Assessment for Mitigation of Spectrum Leakage
}

\author{
Dah-Jing Jwo ${ }^{1, *}$, I-Hua $\mathrm{Wu}^{2}$ and Yi Chang ${ }^{1}$ \\ ${ }^{1}$ Department of Communications, Navigation and Control Engineering, National Taiwan Ocean University \\ 2 Pei-Ning Rd., Keelung 202, Taiwan \\ ${ }^{2}$ Bison Electronics, Inc., Neihu District, Taipei 114, Taiwan
}

\begin{abstract}
This paper investigates the windowing design and performance assessment for mitigation of spectral leakage. A pretreatment method to reduce the spectral leakage is developed. In addition to selecting appropriate window functions, the Welch method is introduced. Windowing is implemented by multiplying the input signal with a windowing function. The periodogram technique based on Welch method is capable of providing good resolution if data length samples are selected optimally. Windowing amplitude modulates the input signal so that the spectral leakage is evened out. Thus, windowing reduces the amplitude of the samples at the beginning and end of the window, altering leakage. The influence of various window functions on the Fourier transform spectrum of the signals was discussed, and the characteristics and functions of various window functions were explained. In addition, we compared the differences in the influence of different data lengths on spectral resolution and noise levels caused by the traditional power spectrum estimation and various window-function-based Welch power spectrum estimations.
\end{abstract}

\section{Introduction}

The spectrum analysis [1-4] is a critical concern in many military and civilian applications, such as performance test of electric power systems and communication systems, detection of mechanical vibration, testing the linearity of components.

Anti-jamming techniques [5,6] have been adopted to enhance the reliability and effectivity of Global Navigation Satellite System (GNSS). As a technique based upon Capon works and Neyman-Pearson theory, the amplitude domain processing (ADP) is an antijamming method to suppress multitone continuous wave, sweep continuous wave and narrowband interference without convergence problems, and therefore has been widely utilized to the GNSS receivers. Using ADP in frequency domain (FADP) will distort useful navigation signal while suppressing interference. FADP filter possesses superior performance, while induces some problems, such as the spectrum leakage of the frequency domain.

To relieve the performance deterioration, the spectral leakage suppression algorithms [6,7] for the DFT-based spectrum analysis is extensively studied, such as digital filtering, high-order spectral analysis, leakage compensation in frequency domain and weighting the time samples by a suitable window function. Window function method is the most widely used. However, window functions are varied and have different characteristics. How to choose suitable window function for different applications requires professional knowledge. Due to computer capacity and limitations on processing time, only limited time samples can actually be processed during spectrum analysis and data processing of signals, i.e., the original signals need to be cut off, which induces leakage errors. In spread spectrum system, frequency-domain interference suppression algorithms always use data windows to reduce the spectral leakage associated with truncation, and employ overlap to lessen the SNR degradation due to windowing.

Direct truncation equals rectangular window shaping. Rectangular time window has narrow main lobe and wide side lobe with small main lobe to side lobe ratio, which results in serious side lobe leakage. A method is proposed to mitigation the influence of spectral leakage of the Fourier transform spectrum by selecting appropriate window functions. Some windows with narrow side lobe, such as Blackman, Hanning, and Kaiser windows are usually employed in engineering instead of the rectangular one.

Periodogram is a method of estimating the autocorrelation of finite length of a signal, which possesses limited ability to produce accurate power spectrum estimation. The nonparametric Welch method $[8,9]$ is an improvement on the periodogram spectrum estimation method where signal to noise ratio is high and reduces noise in the estimated power spectra for reducing the frequency resolution.

This paper is organized as follows. In Section 2, preliminary background on the Fourier transform is reviewed, including the discrete Fourier transform, and

Corresponding author: djjwo@ mail.ntou.edu.tw 
the fast Fourier transform. The spectrum leakage is introduced in Section 3. In Section 4, windowing functions for spectrum leakage suppression is discussed. In Section 5, simulation experiments are carried out to evaluate the performance for various designs. Conclusions are given in Section 6.

\section{Fourier transform}

The Fourier transform is important in engineering, mathematics, and the physical sciences. The Fourier transform is a generalization of the complex Fourier series, defined by

$$
\begin{gathered}
F(\omega)=\int_{-\infty}^{\infty} f(t) e^{-j \omega t} d t \\
f(t)=\frac{1}{2 \pi} \int_{-\infty}^{\infty} F(\omega) e^{j \omega t} d \omega
\end{gathered}
$$

The discrete counterpart of the Fourier transform is the Discrete Fourier Transform (DFT). The DFT is ubiquitous in digital signal processing for the spectrum analysis, which can be efficiently realized by fast Fourier transform (FFT).

\subsection{Discrete Fourier transform}

The discrete Fourier transform converts a finite sequence of equally-spaced samples of a function into a same-length sequence of equally-spaced samples of the discrete-time Fourier transform (DTFT), which is a complex-valued function of frequency. DFT is the equivalent of the continuous Fourier Transform. DTFT is continuous (and periodic), and the DFT provides discrete samples of one cycle. If the original sequence is one cycle of a periodic function, the DFT provides all the non-zero values of one DTFT cycle. The DTFT foe the time domain signal $x[n]$ is given by:

$$
X[\Omega]=\operatorname{DTF}(x[n])=\sum_{-\infty}^{\infty} x[n] e^{-j n \Omega}
$$

We could regard each sample $x[n]$ as windowing function

$$
w_{R}[n]=\left\{\begin{array}{l}
1, n=0,1,2, \mathrm{~L}, N-1 \\
0, \quad \text { otherwise }
\end{array}\right.
$$

Spectrum signal sample is

$$
x_{N}[n]=x[n] w_{R}[n]=\left\{\begin{array}{l}
1, n=0,1,2, \mathrm{~L}, N-1 \\
0, \quad \text { otherwise }
\end{array}\right.
$$

Here we definition signal $\mathrm{N}$ of $x_{N}[n]$ is complete signal. The DFT is the sampled Fourier Transform and therefore does not contain all frequencies forming an image, but only a set of samples which is large enough to fully describe the spatial domain image.

\subsection{Fast Fourier transform}

As the efficient calculation of DFT, the fast Fourier transform (FFT) has been widely used for many applications. The implementations of DFT usually employ efficient fast Fourier transform algorithms, so that the terms FFT and DFT are often used interchangeably. Table 1 provides comparison of workload for DFT and FFT.

Table 1. Comparison of workload for DFT and FFT.

\begin{tabular}{|c|c|c|}
\hline $\mathbf{N}$ & DFT & FFT \\
\hline 2 & 4 & 1 \\
\hline 4 & 16 & 4 \\
\hline 8 & 64 & 12 \\
\hline 16 & 256 & 32 \\
\hline 32 & 1024 & 80 \\
\hline 256 & 4096 & 192 \\
\hline
\end{tabular}

\section{Spectrum leakage}

Spectral leakage is a problem that arises in the digital processing of signals. Signal in practical is always limited in time domain, namely truncated by time window, which results in spectrum leakage of signal. Leakage causes the signal levels to be reduced and redistributed over a broad frequency range, which is important for implementing digital signal processing properly.

The Fourier transform of a function of time, $x(t)$ is a complex-valued function of frequency, and $\mathrm{X}(\mathrm{f})$ is referred to as a frequency spectrum. The flow charrt of the DFT calculation is shown as in Fig. 1. Direct truncation equals rectangular window shaping. Rectangular time window has narrow main lobe and wide side lobe with small main lobe to side lobe ratio, which results in serious side lobe leakage. Fig. 2 shows the discrete spectrum due to signal windowing truncation.

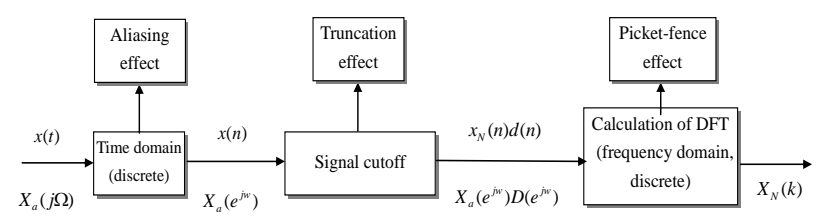

Fig. 1. Flow chart of the DFT calculation.

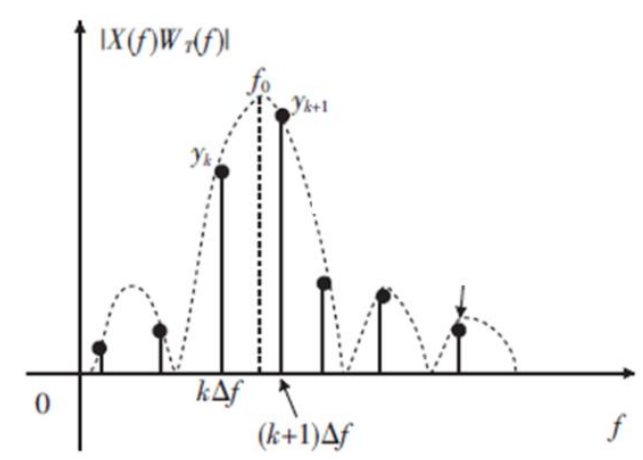

Fig. 2. Discrete spectrum due to signal windowing truncation. 


\subsection{Spectrum leakage suppression - periodic truncation}

The sampling signal length is not integer multiple of the signal period length, and through the Fast Fourier transform (FFT), the sampling signal is going to make larger distortion, therefore it makes many unpredictable spurious components and spectral leakage in discrete spectrum. In conclusion, when signal period is $T_{0}$ and sampling period is $T_{s}$ and sampling point is $N$, it is chosen to satisfy the relation

$$
L T_{0} / T_{s}=N
$$

The FFT algorithm takes the small amount of captured data and repeats it, in order to perform the Fourier Transform and produce a frequency spectrum. In this case, the signal is periodic, and the resulting frequency spectrum will have no leakage. Since most of measured signals will possess non-periodic signals, the problem is how to minimize leakage in these cases. Figs. 3 and 4 show magnitude (in $\mathrm{dB}$ ) of the DFT spectrum using a periodic and non-periodic truncation, respectively.

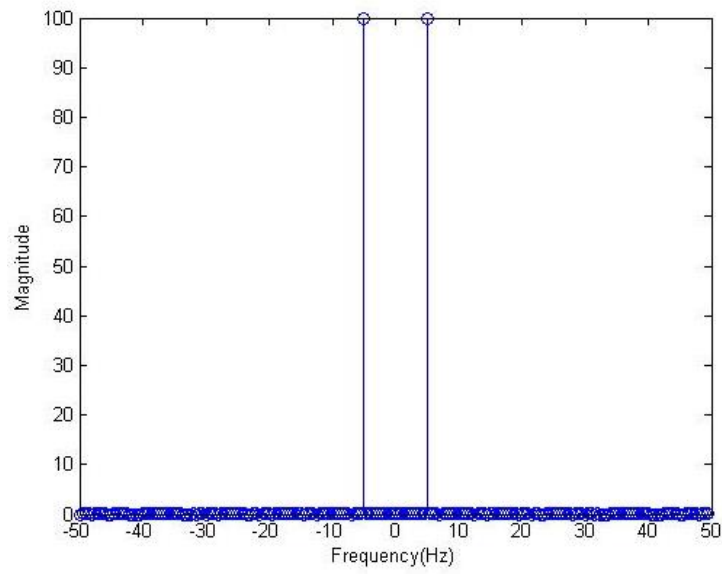

Fig. 3. Magnitude (in $d B$ ) of the DFT spectrum using a periodic truncation.

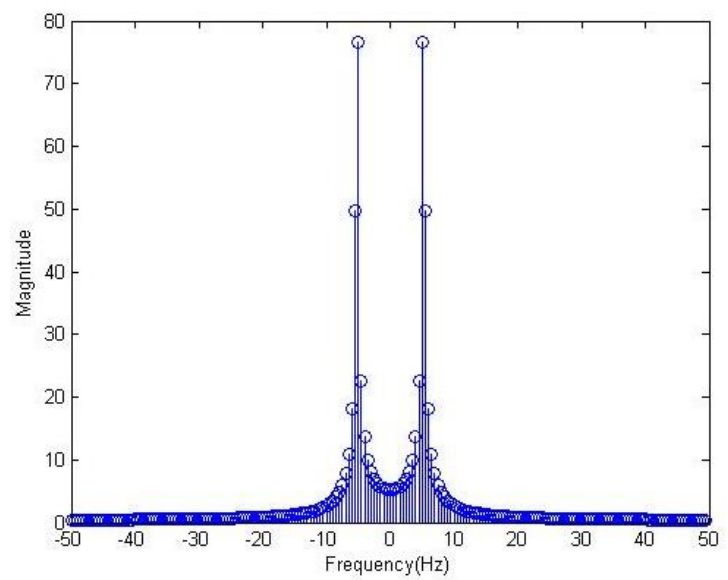

Fig. 4. Magnitude (in $\mathrm{dB}$ ) of the DFT spectrum using a nonperiodic truncation.

\subsection{Spectrum leakage suppression - enlarging the data length in windowing function}

Windowing is implemented by multiplying the input signal with a windowing function. Windowing amplitude modulates the input signal so that the spectral leakage is evened out. Thus, windowing reduces the amplitude of the samples at the beginning and end of the window, altering leakage. An input signal can be of any number of dimensions and can be complex. For complex-values a complex multiplication is required.

As data length increase, the rate of fluctuation in this is also increase. The variance of the periodogram is reduced by applying a window to the autocorrelation estimate to decrease the contribution of unreliable estimates to the periodogram. Increasing the width of window function is equivalent to enlarge the length of segment with large main lobe to side lobe ratio, which results in less side lobe leakage. Figs. 5 and 6 show the magnitude (in $\mathrm{dB}$ ) of the DFT spectrum using a simple length-32 $(\mathrm{N}=32)$ and length-64 $(\mathrm{N}=64)$, truncation windows, respectively. The quality of the estimate increases as the length $\mathrm{N}$ of the data increase.

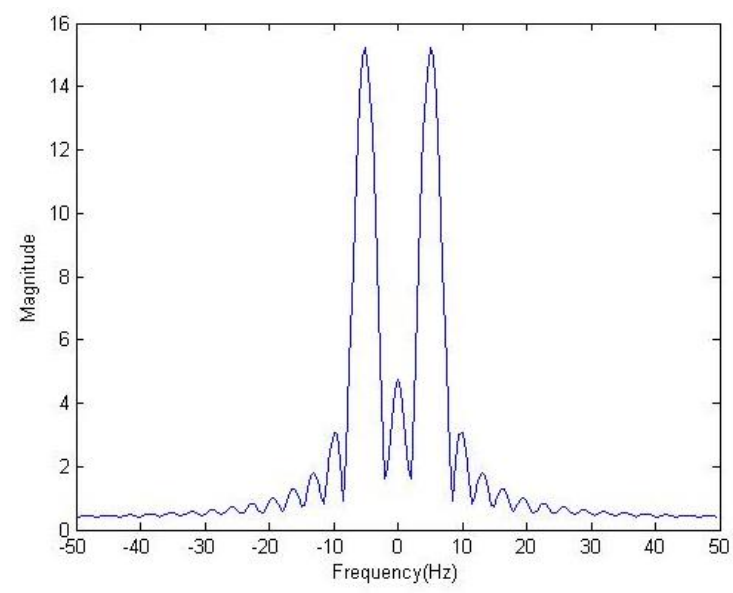

Fig. 5. Magnitude (in $\mathrm{dB}$ ) of the DFT spectrum using a simple length-32 truncation window $(\mathrm{N}=32)$.

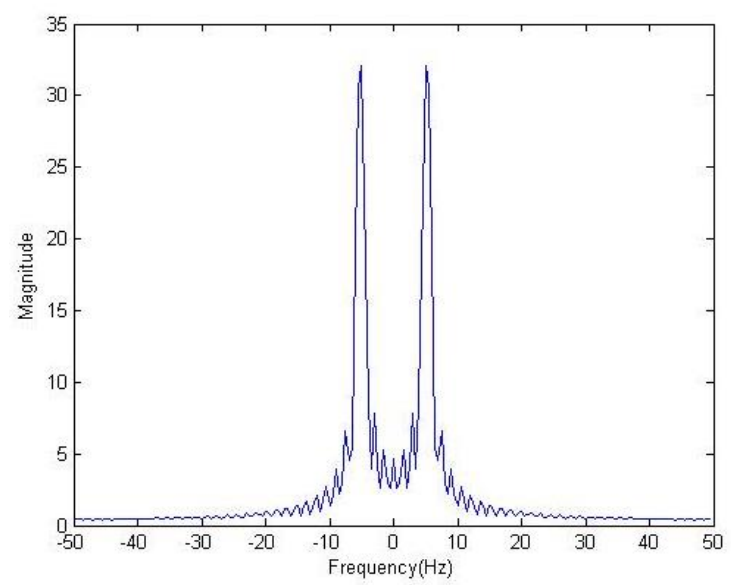

Fig. 6. Magnitude (in $\mathrm{dB}$ ) of the DFT spectrum using a simple length-64 truncation window $(\mathrm{N}=64)$. 


\section{Windowing functions for spectrum leakage suppression}

The method of windowing functions is most popular method for inhibition of spectral leakage. Windowing functions are also called tapering functions or apodization functions. Many different window functions have been developed for truncating and shaping a length$\mathrm{N}$ signal segment for spectral analysis. It inhibits the spectral leakage by cutting the signal when the period is delay and two-side is continuous. There are many common windowing functions. The special techniques like window function and rectangular, Hanning, Blackman, and Kaiser window to extract the unwanted noise from the signal.

\subsection{Rectangular window}

A function that is constant inside the interval and zero elsewhere is called a rectangular window. Direct truncation equals rectangular window shaping. The simple truncation window has a periodic sinc DTFT. It has the narrowest main-lobe width, $2 \pi / \mathrm{N}$ at the $-3 \mathrm{~dB}$ level and $4 \pi / \mathrm{N}$ between the two zeros surrounding the main lobe, of the common window functions, but also the largest side-lobe peak, at about $-13 \mathrm{~dB}$. The sidelobes also taper off relatively slowly. The truncation to a length $M-1$ is equivalent to multiplying the signal by a rectangular window. Expression of normal rectangular time window is defined as

$$
w(n)=R_{N}(n) \begin{cases}1 & 0 \leq n \leq N-1 \\ 0 & \text { Otherwise }\end{cases}
$$

The frequency domain can be expressed as

$$
W\left(e^{j \omega}\right)=\frac{\sin \left(\frac{\omega N}{2}\right)}{\sin \left(\frac{\omega}{2}\right)} e^{-j \omega\left(\frac{N-1}{2}\right)}
$$

Fig. 7 gives the time domain amplitude and magnitude spectrum with rectangular window. The rectangular window has good resolution characteristics for sinusoids of comparable strength.

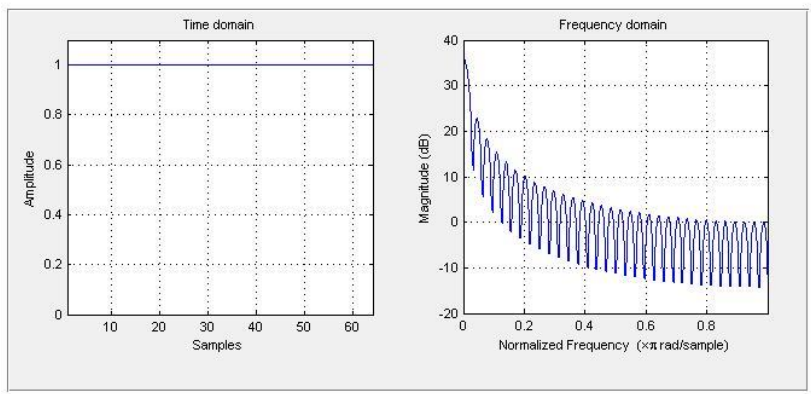

Fig. 7. Time domain amplitude and magnitude spectrum with rectangular window.

Rectangular time window has narrow main lobe and wide side lobe with small main lobe to side lobe ratio, which results in serious side lobe leakage. The minimum stopband attenuation is $21 \mathrm{~dB}$.

\subsection{Blackman window}

The Blackman method is used to reduce variance of the estimator. If we compare Blackman window with other windows there is better improvement in stopband attenuation. Blackman window has good characteristics for audio work. The Blackman window in time domain is defined as

$$
w(n)=\left(0.42-0.5 \cos \left(\frac{2 \pi n}{N-1}\right)+0.08 \cos \left(\frac{4 \pi n}{N-1}\right)\right) R_{N}(n)
$$

The frequency domain is given by

$$
\begin{aligned}
W\left(e^{j \omega}\right) & =0.42 W_{R}(\omega)-0.25\left[W_{R}\left(\omega-\frac{2 \pi}{N-1}\right)+W_{R}\left(\omega+\frac{2 \pi}{N-1}\right)\right] \\
& +0.04\left[W_{R}\left(\omega-\frac{4 \pi}{N-1}\right)+W_{R}\left(\omega+\frac{4 \pi}{N-1}\right)\right]
\end{aligned}
$$

Fig. 8 shows the time domain amplitude and magnitude spectrum with Blackman window.

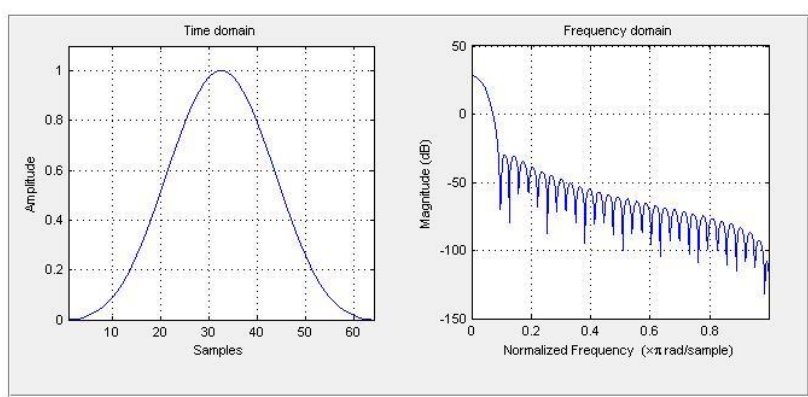

Fig. 8. Time domain amplitude and magnitude spectrum with Blackman window.

The maximum side lobe for the Blackman window is $57 \mathrm{~dB}$ lower than main lobe, while which is three times as that of rectangular window, and the minimum stopband attenuation is $74 \mathrm{~dB}$.

\subsection{Hanning window}

The Hanning window (also called the Hanning window) is also known as a raised-cosine window due to its shape and form. The advantage of using Hanning Window method is that it reduces the side lobes. It has good frequency resolution over other window method like rectangular window. The Hann window takes the form:

$$
w(n)=0.5\left(1-\cos \left(2 \pi \frac{n}{N+1}\right)\right) R_{N}(n) \quad n=1,2, \Lambda, N
$$

The frequency domain is defined as

$$
W\left(e^{j \omega}\right)=0.58 W_{R}(\omega)-0.25\left[W_{R}\left(\omega-\frac{2 \pi}{N-1}\right)+W_{R}\left(\omega+\frac{2 \pi}{N-1}\right)\right]
$$

It has a main-lobe width considerably larger than the rectangular window, but the largest side-lobe peak is much lower, at about $-31.5 \mathrm{~dB}$. The side-lobes also taper off much faster. For a given length, this window is worse than the boxcar window at separating closely-spaced spectral components of similar magnitude, but better for 
identifying smaller-magnitude components at a greater distance from the larger components. It has a main-lobe width (about $3 \pi / N$ at the $-3 \mathrm{~dB}$ level and $8 \pi / N$ between the two zeros surrounding the main lobe. Fig. 9 provides the time domain amplitude and magnitude spectrum with Hanning window.

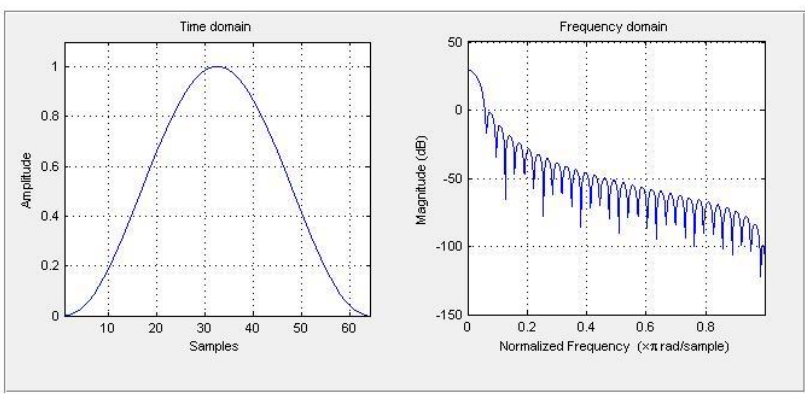

Fig. 9. Time domain amplitude and magnitude spectrum with Hanning window.

The Hamming window is use to minimize the maximum nearest side lobes, which has a form similar to the Hann window but with slightly different constants. Since it is composed of the same Fourier series harmonics as the Hann window, it has a similar mainlobe width, but the largest side-lobe peak is much lower, at about $-42.5 \mathrm{~dB}$. However, the side-lobes also taper off much more slowly than with the Hann window. For a given length, the Hamming window is better than the Hann windows at separating a small component relatively near to a large component, but worse than the Hann for identifying very small components at considerable frequency separation.

\subsection{Kaiser window}

The Kaiser window is a simple approximation of the DPSS window using Bessel functions, discovered by James Kaiser. As compared to the other window functions the Kaiser Window has maximum attenuation according to the given width of the main lobe. That is why it is also known as optimal window. The Kaiser Window function can be written:

$$
w(n)=\frac{I_{0}\left[\beta \sqrt{1-\left(1-\frac{2 n}{N-1}\right)^{2}}\right]}{I_{0}(\beta)} \quad 0 \leq n \leq N-1
$$

where $I_{0}(\beta)$ is the zero-th order modified Bessel function of the first kind. The variable parameter $\beta$ determines the tradeoff between main lobe width and side lobe levels of the spectral leakage pattern.

$$
\beta=\left\{\begin{array}{cl}
0.1102(\alpha-8.7) & \alpha>50 \\
0.5482(\alpha-21)^{0.4}+0.07886(\alpha-21) & 21 \leq \alpha \leq 50 \\
0 & \alpha<21
\end{array}\right.
$$

Fig. 10 gives the time domain amplitude and magnitude spectrum with Kaiser window.
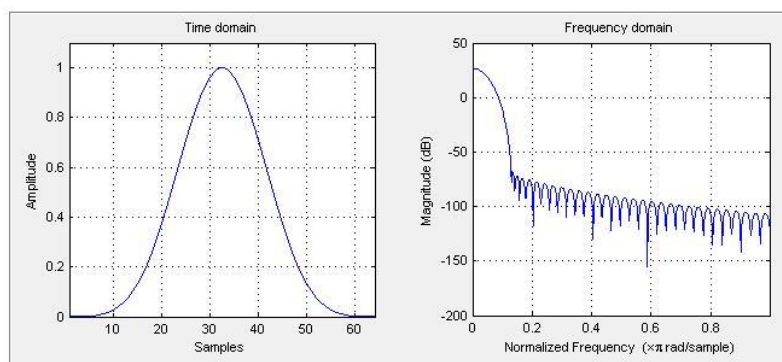

Fig. 10. Time domain amplitude and magnitude spectrum with Kaiser window.

\subsection{The Welch method}

The periodogram technique based on Welch method is capable of providing good resolution if data length samples are selected optimally. Welch's method is also called the periodogram method for estimating power spectra. The approach is carried out by dividing the time signal into successive blocks, forming the periodogram for each block, and averaging. As a non-parametric estimator of the power spectral density (PSD) of a random signal, the periodogram is not consistent due to the fact that its variance does not converge towards zero even when the length of the random signal is increased towards infinity. The Welch method is usually employed to overcome this problem, based on the following procedure: (1) split the random signal into segments; (2) estimate the PSD for each segment; and (3) average over these local estimates. The averaging reduces the variance of the estimated PSD. The Welch's method is a generalization using windowed overlapping segments.

A good resolution in power spectrum estimation (PSE) may be achieved by using optimum size of data sample. PSE has been performed using various windowing functions with the Welch method. Welch method is nonparametric method that include the periodogram that possesses the advantage of possible implementation using the fast Fourier Transform. In this section, the Welch method gives the result on power spectrum estimation with different windows. In DSP, Welch method is used to find the PSD of a signal with reducing the effect of noise.

A classical periodogram approach is an estimate of the spectral density of a signal, as shown in Fig. 11. Fig. 12 provides the flowchart of Welch method. An input signal can be of any number of dimensions and can be complex.

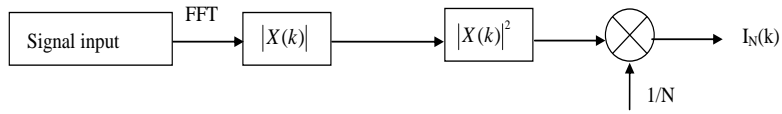

Fig. 11. Folw chart of the classical periodogram approach.

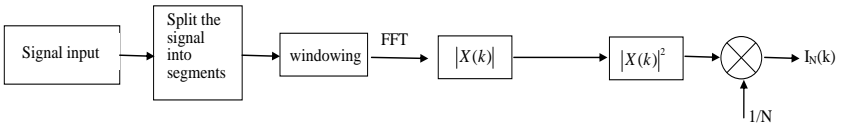

Fig. 12. Folw chart of the Welch method. 


\section{Results and Discussion}

To investigate the capability of various window function for inhibition of spectral leakage, various windowing functions incorporated into the Welch power spectrum is carried out. Some results based on the numerical simulation are provided.

\subsection{Spectra of a sine wave using various windowing functions}

In the first test example, the continuous sinusoid signal $x(t)=\sin (2 \pi f t)$, is employed, where $f=5.2 \mathrm{~Hz}$, and the sampling frequency is $f_{s}=100 \mathrm{~Hz}$, Truncate length $\mathrm{N}=$ 64. The rectangular window has admirable resolution characteristics for sinusoids of comparable strength.

The original time domain sinusoid signal and its magnitude spectrum are shown as in Fig. 13. Fig. 14 provides the rectangle windowed sinusoid and its magnitude spectrum. Figs. 15-17 provide the time domain signal and the corresponding magnitude spectra using the Blackman, Hanning, and Kaiser window, respectively.
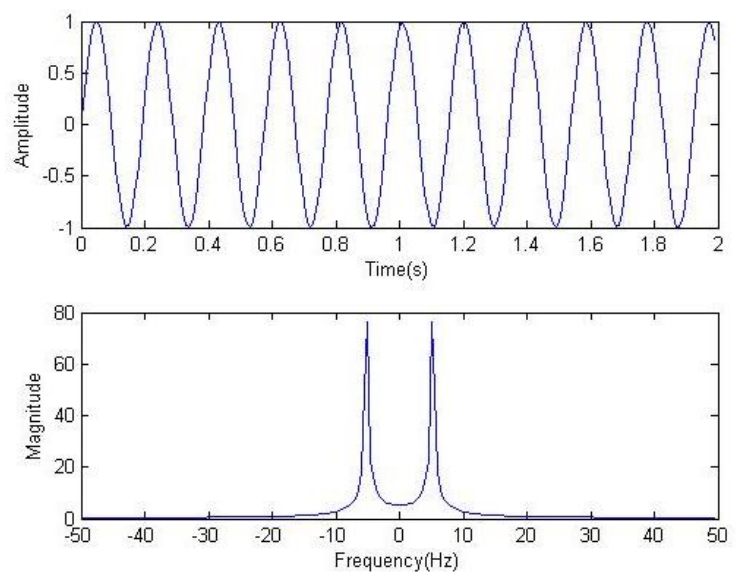

Fig. 13. Original time domain sinusoid signal and its magnitude spectrum.
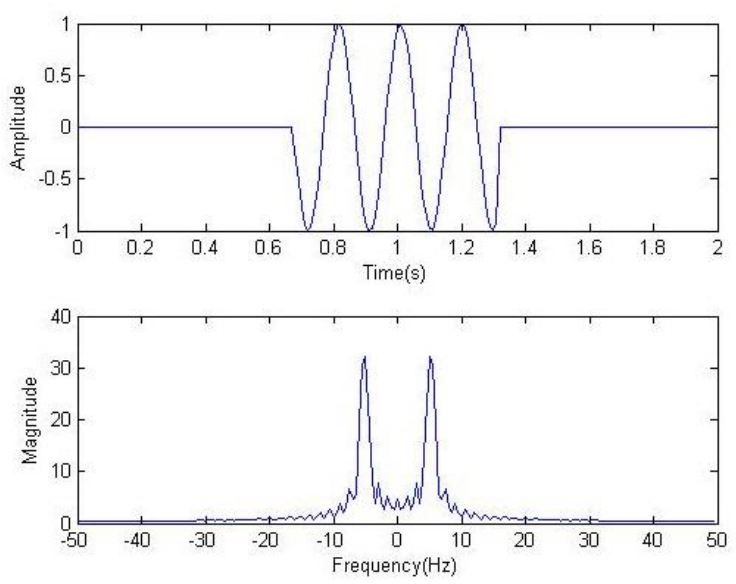

Fig. 14. The rectangle windowed sinusoid and its magnitude spectrum.
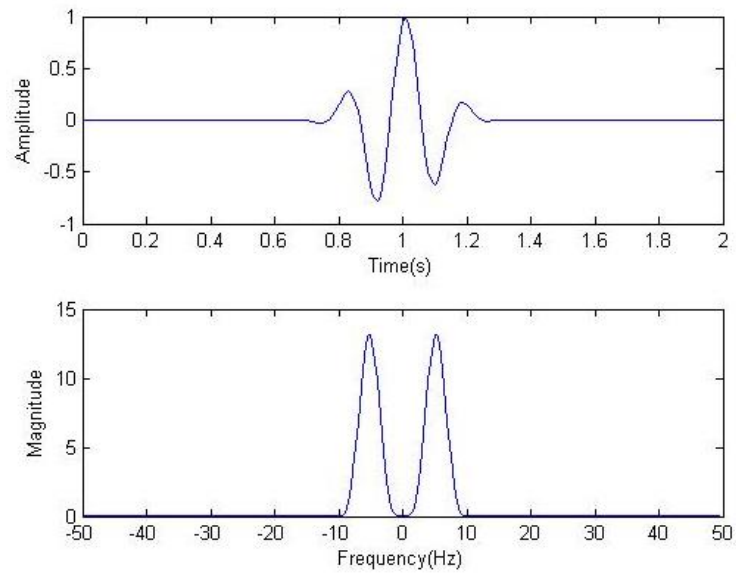

Fig. 15. Time domain signal with Blackman window and its magnitude spectrum.
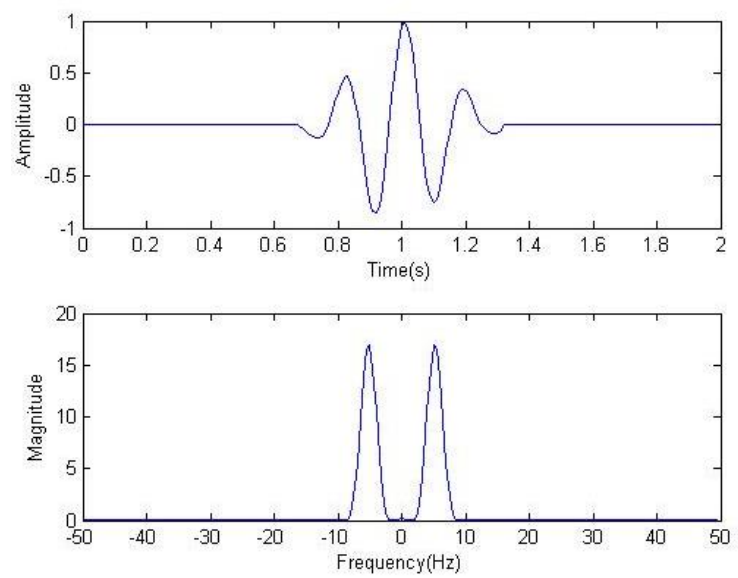

Fig. 16. Time domain signal with Hanning window and its magnitude spectrum.
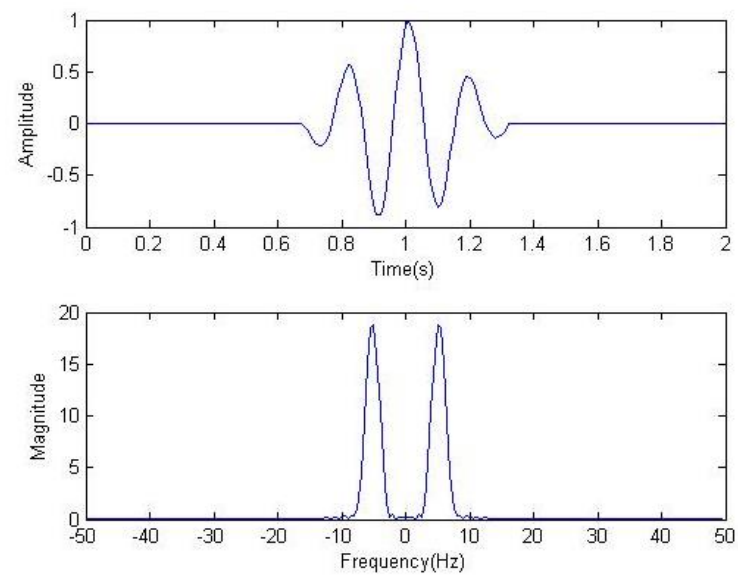

Fig. 17. Time domain signal with Kaiser window and its magnitude spectrum.

\section{2 spectrum estimation using Welch method with various windowing functions}

The simulation employed a signal is a sine signal with frequency $300 \mathrm{~Hz}$ plus another sine signal with frequency $500 \mathrm{~Hz}$ as the input. A Gaussian-white sequence is added into the signal, where the signal-to-noise ratio is $10 \mathrm{~dB}$. The sampling frequency is $3000 \mathrm{~Hz}$ with the number of sampling points 3000. The data length of FFT is 512 . 
The characteristics of window function are analyzed from spectrum resolution and noise level. Simulation based on the periodogram method and then the Welch method using the rectangular, Blackman, Hanning window, and Kaiser windows (all with length 511) are performed for power spectrum estimation.

The selection and use of window function in Welch algorithm are analyzed. Power spectrum estimation using the periodogram is shown as in Fig. 18. Figs. 1922 provide the power spectrum magnitude using Welch method with rectangular, Blackman, Hanning, and Kaiser windows, respectively. Comparison of Spectral estimation using various approaches is summarized in Table 2.

The rectangular window has the clear peak in the graph showing the power spectrum estimation. The spectral resolution of rectangular window and Kaiser window is higher, but the noise level near the signal frequency is higher. It is considered that both rectangular window and Kaiser window are suitable for high precision spectrum estimation of signals with high signal to noise ratio. Hanning window has better effect on spectrum leakage suppression, but the frequency resolution is relatively low, suitable for general frequency estimation of signals with low signal to noise ratio.

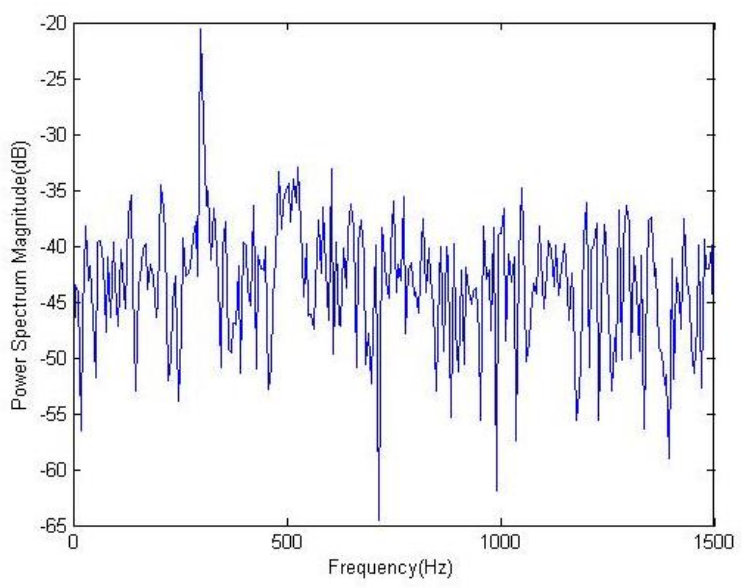

Fig. 18. Power spectrum estimation using the periodogram.

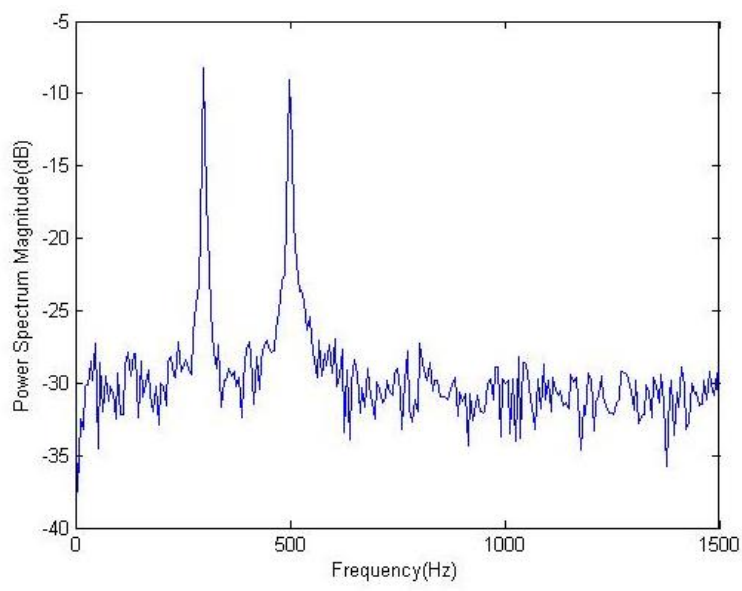

Fig. 19. Power spectrum magnitude using Welch method with rectangular window.

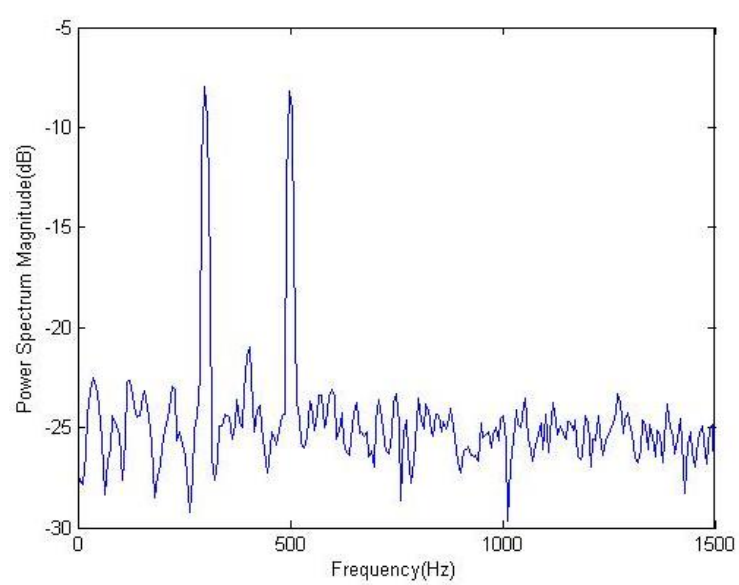

Fig. 20. Power spectrum magnitude using Welch method with Blackman window.

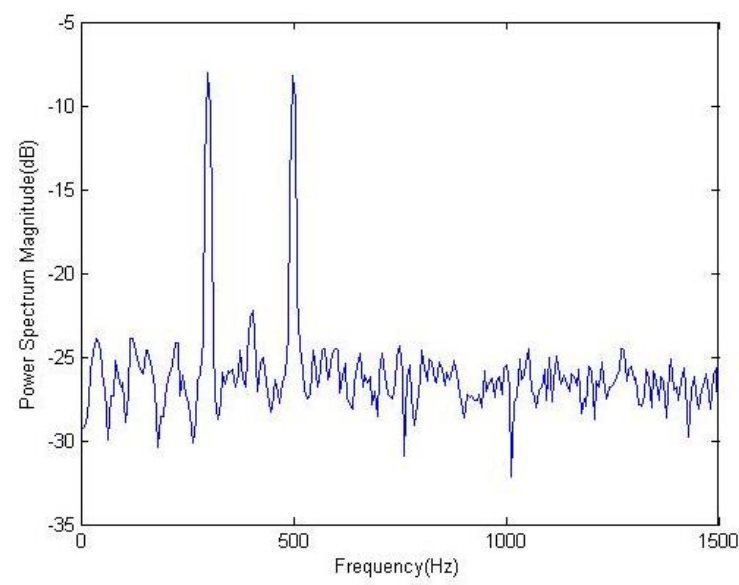

Fig. 21. Power spectrum magnitude using Welch method with Hanning window.

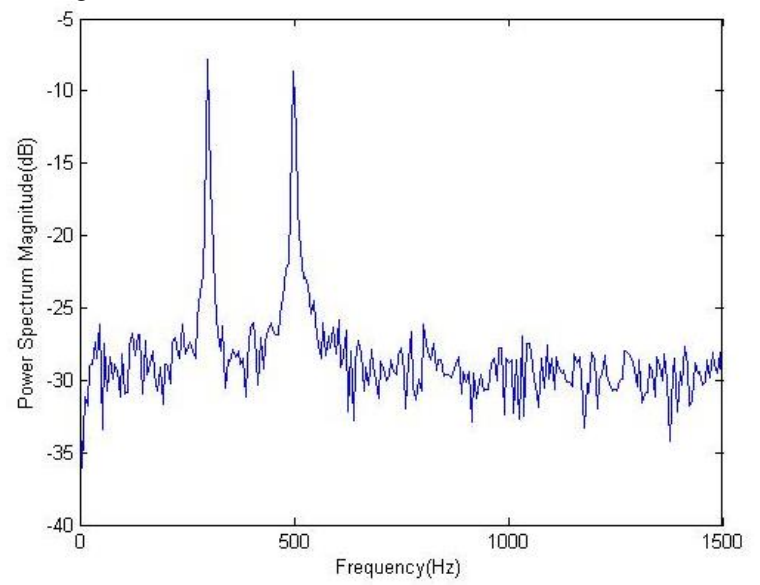

Fig. 22. Power spectrum magnitude using Welch method with Kaiser window.

\section{Conclusions}

This paper investigates the spectrum leakage suppression algorithm. The power spectrum estimation has been performed using various windowing functions with the Welch method. 
Resolution is the ability to discriminate spectral feature and is a key concept on the analysis of spectral estimator performance. Estimation with low spectrum resolution causes the signal easily disturbed by the noise, leading to the failure of the detection. The performance of DFT method inherently suffers from the spectral leakage, which is caused by the non-coherent sampling in the practical acquisition system. The superiority on spectrum leakage suppression algorithm is verified by comparing with the traditional spectrum analysis based on the DFT with windowing. The quality of the estimate increases as the length $\mathrm{N}$ of the data increase.

Windowing amplitude modulates the input signal so that the spectral leakage is evened out. The windowing functions in this paper including the rectangular, Blackman, Hanning, and Kaiser windows have been employed to extract the unwanted noise from the signal. The effect of windowing functions on power spectral density is investigated by applying the Welch method. The Welch algorithm splits the random signal into segments before DFT to reduce the spectral leakage, which used a modified version of Bartlett method in which the portion of the series contributing to each periodogram are allowed to overlap. The Welch method gives the result on power spectrum estimation with different windowing functions in the window method to estimate the power spectral density of a signal, with less effect caused by noise.

Table 2. Comparison of Spectral estimation using various approaches.

\begin{tabular}{|c|c|c|c|}
\hline $\begin{array}{c}\text { Windowing } \\
\text { function }\end{array}$ & $\begin{array}{c}\text { Average } \\
\text { spectral } \\
\text { error (Hz) }\end{array}$ & $\begin{array}{c}\text { Average } \\
\text { noise } \\
\text { power (dB) }\end{array}$ & $\begin{array}{c}\text { spectral leakage } \\
\text { mean power } \\
\text { (dB) }\end{array}$ \\
\hline $\begin{array}{c}\text { Rectangular } \\
\text { window }\end{array}$ & \pm 1.3672 & -29.4467 & -23.4521 \\
\hline $\begin{array}{c}\text { Blackman } \\
\text { window }\end{array}$ & \pm 2.4038 & -25.1630 & -25.9652 \\
\hline $\begin{array}{c}\text { Hanning } \\
\text { window }\end{array}$ & \pm 2.8646 & -26.1223 & -26.2241 \\
\hline $\begin{array}{c}\text { Kaiser } \\
\text { window }\end{array}$ & \pm 1.4625 & -28.2806 & -25.2102 \\
\hline
\end{tabular}

\section{Acknowledgements}

Funding for this work was provided by the Ministry of Science and Technology of the Republic of China under grant number MOST 107-2221-E-019-025

\section{References}

1. C. L. Phillips and H. T. Nagle, Digital Control System Analysis and Design, 3d ed. Upper Saddle River, NJ: Prentice Hall, (1996).

2. S. S. Soliman and M. D. Srinath, Continuous and Discrete Signals and Systems, 2d ed. Upper Saddle River, NJ: Prentice Hall, (1997).

3. R. E. Ziemer, W. H. Tranter, and S. R. Fannin, Signals and System Continuous and Discrete, 4th ed. (1998).
4. C. L. Phillips and H. T. Nagle, Digital Control System Analysis and Design, Upper Saddle River, NJ: Prentice Hall (1996).

5. T. Zhang, et. al, Effect of Frequency Domain AntiJamming Filter on Satellite Navigation Signal Tracking Performance, China Satellite Navigation Conference (CSNC, 2013), Lecture Notes in Electrical Engineering 243, Chapter 46, J. Sun et al. (eds.), pp. 507-516.

6. J. Li, et. al, Effects of Spectrum Leakage on Frequency Domain Anti-jamming Performance, China Satellite Navigation Conference (CSNC, 2016), Volume I, Lecture Notes in Electrical Engineering 388, pp.631-642.

7. Q. Wang, X. Yan, Y. Ren, Spectrum Leakage Suppression for Multi-Frequency Signal based on DFT, IEEE 13th International Conference on Electronic Measurement \& Instruments (2017).

8. P. D. Welch, The Use of Fast Fourier Transform for the Estimation of Power Spectra: A Method Based on Time Averaging Over Short Modified Periodograms, IEEE Trans. Audio Electroacoustic (1970).

9. H. R. Gupta, R. Mehra, Power Spectrum Estimation using Welch Method for Various Window Techniques, International Journal of Scientific Research Engineering \& Technology (2013). 